\begin{tabular}{l|l|}
\hline $\begin{array}{l}\text { 2. To: (Receiving Organization) } \\
\text { Distribution }\end{array}$ & $\begin{array}{l}\text { 3. From: (Originating Organization) } \\
\text { J. D. Galbraith, NHC }\end{array}$ \\
$\begin{array}{l}\text { 5. Proj./Prog./Dept./Div.: } \\
\text { TWRS }\end{array}$ & $\begin{array}{l}\text { 6. Design Authority/ Design Agent/Cog } \\
\text { Engr.: }\end{array}$ \\
\hline
\end{tabular}

8, Originator Remarks:

For approval and release.

11. Receiver Remarks: 11A. Design Baseline Document? [] Yes [X] No

4. Related EDT No.:

NA

7. Purchase Order No.:

NA

9. Equip./Component No.:

NA

10. System/Bldg./Facility:

NA

12. Major Assm. Owg. No.:

NA

13. Permit/Pernit Application No.: NA

14. Required Response Date:

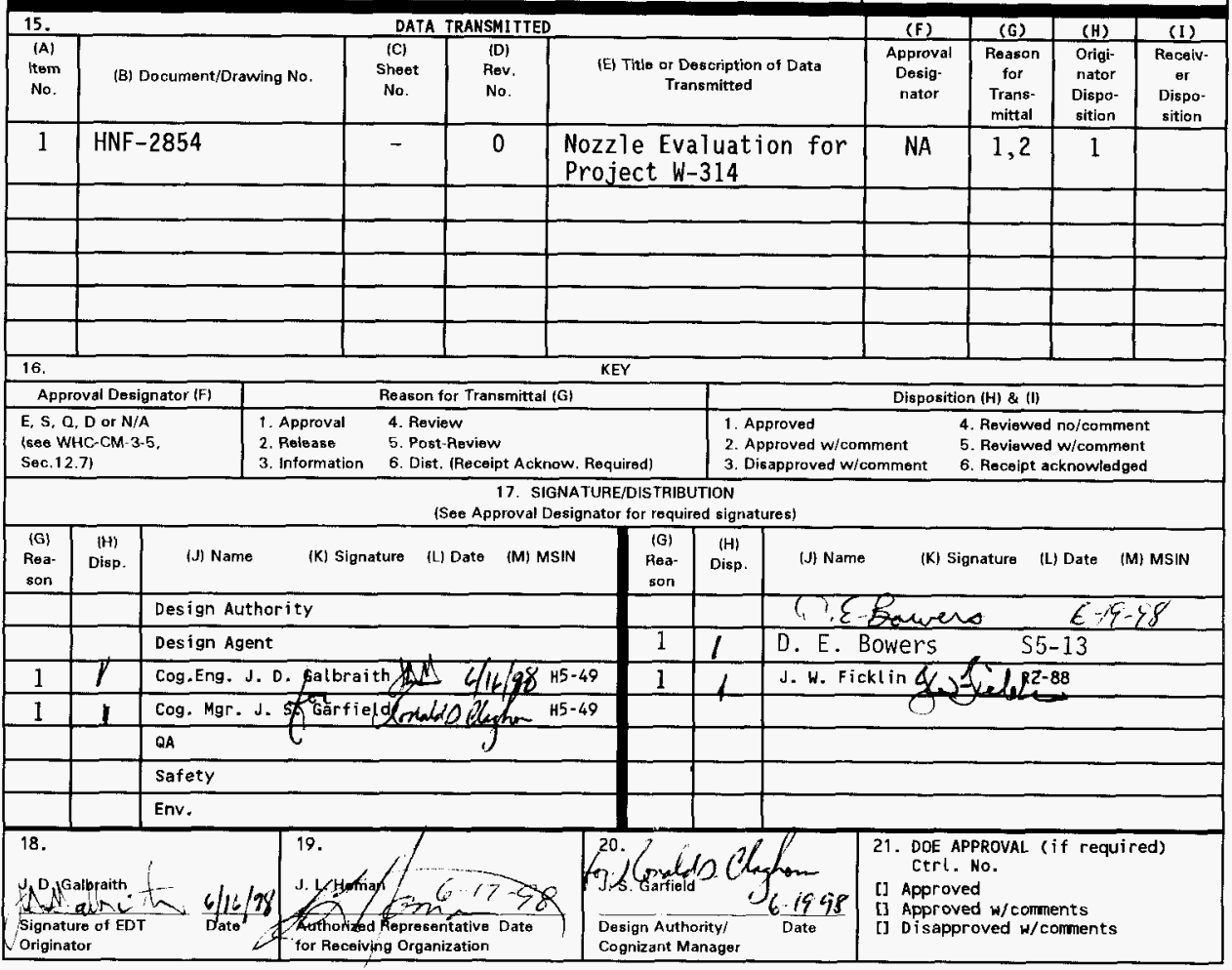

BD-7400-172-2 (05/96) GEF097 
HNF-2854, Rev. 0

\section{Nozzle Evaluation for Project W-314}

J. D. Galbraith and J. W. Fickl in (LMHC)

Numatec Hanford Corporation, Richland, WA 99352

U.S. Department of Energy Contract DE-AC06-96RL13200

EDT/ECN: $622723 \quad$ UC: 721

Org Code: 8C451 Charge Code: D2D77

B\&R Code: EW3130010 Total Pages: 21

Key Words: nozzle, W-314

Abstract: Revisions to the waste transfer system piping to be implemented by Project $W-314$ will eliminate the need to access a majority of interfarm jumper connections associated with specific process pits. Additionally, connections that formerly facilitated waste transfers from the Plutonium-Uranium Extraction (PUREX) P1ant are no longer required. This document identified unneeded process pit jumper connections, describes former designated routing, denotes current status (i.e., "open" or "blanked"), and recommends appropriate disposition for a11. Blanking of identified nozzles should be accomplished by Project W-314 upon installation of jumpers and acceptance by Tank Waste Remediation System (TWRS) Tank Farm Operations.

TRADEMARK DISCLAIMER. Reference herein to any specific comercial product, process, or service by trade name, trademark, manufacturer, or otherwise, does not necessarily constitute or imply its endorsement, recormendation, or favoring by the United States Government or any agency thereof or its contractors or subcontractors.

Printed in the United States of America. To obtain copies of this document, contact: Document Control Services, P.O. Box 950, Maitstop H6-08, Richland WA 99352, Phone (509) 372-2420;

Fax (509) 376-4989.
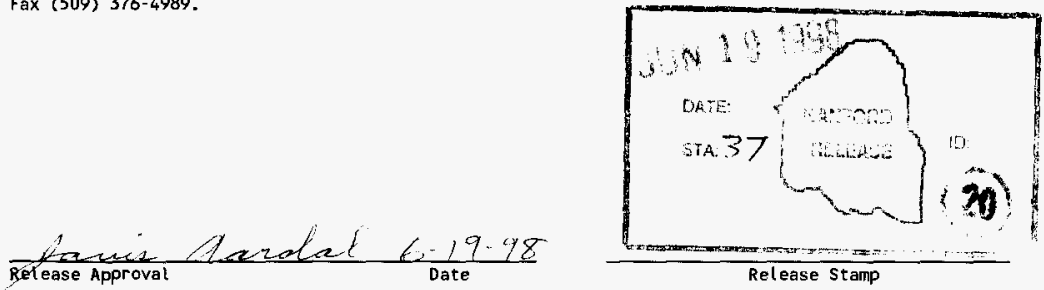

Approved for Public Release 
HNF-2854

Revision 0

\section{NOZZLE EVALUATION FOR PROJECT W-314}

June 1998

J. D. Galbraith

Numatec Hanford Corporation

Richland, Washington.

J. W. Ficklin

Lockheed Martin Hanford Corporation

Richland, Washington

Prepared for

U.S. Department of Energy

Richland, Washington 
HNF-2854

Revision 0

This page intentionally left blank. 
HNF-2854

Revision 0

\section{CONTENTS}

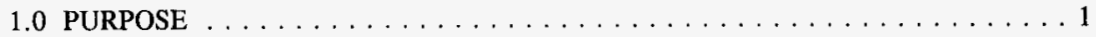

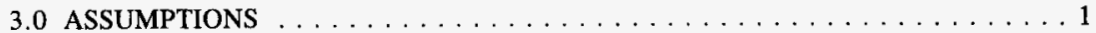

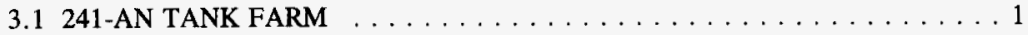

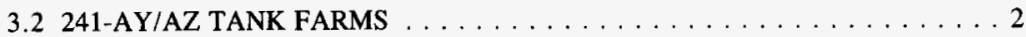

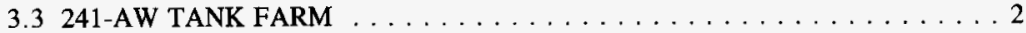

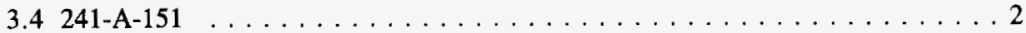

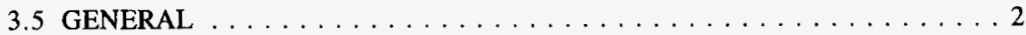

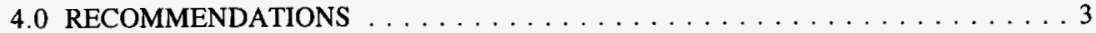

\section{LIST OF TABLES}

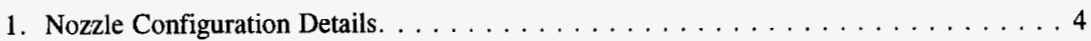

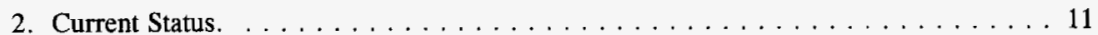


HNF-2854

Revision 0

LIST OF TERMS

PUREX Plutonium-Uranium Extraction

TWRS Tank Waste Remediation System 
HNF-2854

Revision 0

\section{NOZZLE EVALUATION FOR PROJECT W-314}

\subsection{PURPOSE}

Revisions to the waste transfer system piping to be implemented by Project W-314 will eliminate the need to access a majority of interfarm jumper connections associated with specific process pits. Additionally, connections that formerly facilitated waste transfers from the Plutonium-Uranium Extraction (PUREX) Plant are no longer required. This document identifies unneeded process pit nozzle/jumper connections, describes former designated routing, denotes current status (i.e., "open" or "blanked"), and recommends appropriate disposition for all. Blanking of identified nozzles, see Table 1, should be accomplished by Project W-314 upon installation of jumpers and acceptance by Tank Waste Remediation System (TWRS) Tank Farm Operations.

\subsection{SCOPE}

This evaluation addresses identified process pits and post W-314 jumper connections at the following 200 East Tank Farm locations:

241-AN, 241-AY, 241-AZ, 241-AW, and the 241-A-151 Diversion Box.

\subsection{ASSUMPTIONS}

\subsection{1-AN TANK FARM}

- SN lines/nozzles will be connected to support waste retrieval/transfer operations.

- SL lines/nozzles will be used to support delivery of diluent to the installed new transfer pumps. This connection will also provide the ability to flush the SL (2-in.) lines.

- New flush (3-in.) and diluent flush (2-in.) lines will be provided by W-211 (nozzles L-20, 2-in.; L-21, 3-in. in AN-A; R-20, 2-in. in AN-B). 


\subsection{1-AY/AZ TANK FARMS}

- The installation of isolation blanks on existing lines in 241-AZ and 241-AY Tank Farms, eliminates any compelling need for W-314 to enter valve pits 241-AX-A, 241-AX-B, or 241-A-B to install redundant isolation blanks. If additional isolation is desired, TWRS Tank FarmOperations will be responsible for this scope of work.

- At present, the U-4 nozzle in 241-AZ-02A Central Pump Pit is being utilized to facilitate $241-A Z-151$ catch tank pumping. While there is a need to eventually reroute this tank, such change currently has no Operations priority. Since the existing route will continue to be used, there is no recommendation to blank U-4.

\subsection{1-AW TANK FARM}

: SL lines/nozzles will be used to support delivery of diluent to the "new" transfer pump. This connection will also provide the ability to flush the SL (2-in.) lines.

- SL nozzles/lines will also be used to support 242-A Evaporator operations. It is assumed that the 242-A operation will continue to use 241-AW-102 as the feed staging tank and 241-AW-104 and 106 as receivers during the Evaporator campaign/operation. Slurry waste collected in 241-AW-104 and 106 will then be transferred to 241-AN or 241-AP Tank Farms, as space is available. These transfers will use the 3-in. SN transfer lines.

- SN-220 in 241-AW-A valve pit will be used to receive waste transfers from 204-AR via the 241-A-A valve pit by connecting nozzles L-11 (LIQW-702) and L-2 (SN-220). Another option would be to cut/reroute these lines outside of the A-A valve pit and connect the two lines together.

- Jumper connections and nozzles to be blanked (refer to Table 1) currently are not the responsibility of W-314. Project W-454 has the responsibility for providing the jumper configurations identified.

\subsection{1-A-151}

- Nozzles from this diversion box to the 241-AW valve pits should be blanked; however, this activity is currently outside of the W-314 work scope.

\subsection{GENERAL}

- All encasement drain seal piping should be left open. This issue requires further review with TWRS operations and permitting organizations. 
HNF-2854

Revision 0

\subsection{RECOMMENDATIONS}

The rationale behind blanking unneeded nozzles is twofold. First, such action effectively removes unnecessary equipment from service, eliminating any liability inherent to the operation or maintenance of this equipment. Additionally, it is deemed prudent to install process blanks at all unneeded nozzles to preclude the possibility of improper jumper connections and thus mitigate potential for waste transfer misrouting.

Table 1 provides an all-inclusive listing of process pits and respective nozzles as indicated on drawing ES-314E-M40, Rev 8, plus selective nozzles in the 241-A-151 Diversion Box. This table reflects the future disposition of all nozzles listed.

Table 2 provides a listing of process pits and respective nozzles; these are grouped by process pit location. The "Status" column indicates present configuration as documented by one or more of the following sources:

- 272-AW Routing Board (as of May 19, 1998)

- Pit video records

- Pit configuration drawings.

Configurations listed are as depicted via the 272-AW Routing Board, unless otherwise indicated. Information from other sources/contradicting configurations are highlighted by an asterisk (*). 
HNF-2854

Revision 0

Table 1. Nozzle Configuration Details. (7 Sheets)

\begin{tabular}{|c|c|c|c|c|c|}
\hline Nozzle & Size & Pipe \#/ Use & Configuration/Status & Jumper & Blank \\
\hline \multicolumn{6}{|c|}{ Location: Valve Pit AN-A Drawing No.: ES-314E-M40, Rev 7; H-14-20801; H-14-10094 } \\
\hline $\mathrm{L}-1$ & 3-in. & SN-267 & To AN-07A (A) & $\mathrm{X}$ & \\
\hline L-2 & 2 -in. & Spare & $\begin{array}{l}\text { Isolation blank } \\
\text { installed }\end{array}$ & & $\mathrm{X}$ \\
\hline $\mathrm{L}-3$ & 2 -in. & SL-167 & To AN-07A (B) & $\mathrm{X}$ & \\
\hline L-4 & 2 -in. & Spare & $\begin{array}{l}\text { Isolation blank } \\
\text { installed }\end{array}$ & & $\mathbf{X}$ \\
\hline L-5 & 2 -in. & SL-164 & To AN-04A (B) & $\mathrm{x}$ & \\
\hline $\mathrm{L}-7$ & 2 -in. & SL-165 & To AN-05A (B) & $\mathrm{X}$ & \\
\hline $\mathrm{L}-9$ & 2 -in. & SL-166 & To AN-06A (B) & $\mathrm{x}$ & \\
\hline $\mathrm{L}-10$ & 2 -in. & Spare & $\begin{array}{l}\text { Isolation blank } \\
\text { installed }\end{array}$ & & $\mathrm{X}$ \\
\hline $\mathrm{L}-11$ & 3-in. & Spare & $\begin{array}{l}\text { Isolation blank } \\
\text { installed }\end{array}$ & & $\mathrm{X}$ \\
\hline $\mathrm{L}-12$ & 3-in. & Spare & $\begin{array}{l}\text { Isolation blank } \\
\text { installed }\end{array}$ & & $\mathrm{x}$ \\
\hline $\mathrm{L}-13$ & 3-in. & Spare & $\begin{array}{l}\text { Isolation blank } \\
\text { installed }\end{array}$ & & $\mathrm{X}$ \\
\hline $\mathrm{L}-14$ & 3 -in. & SN-266 & To AN-06A (A) & $\mathrm{x}$ & \\
\hline $\mathrm{L}-15$ & 3 -in. & SN-264 & To AN-04A (A) & $\mathrm{X}$ & \\
\hline L-16 & 3-in. & SN-265 & To AN-05A (A) & $\mathrm{X}$ & \\
\hline $\mathrm{L}-17$ & 2-in. & Existing flush & Process blank installed & & $\mathrm{X}$ \\
\hline $\mathrm{L}-18$ & 2-in. & SL-168 & To AN-B (R18) & & $X$ \\
\hline $\mathrm{L}-19$ & 3 -in. & SN-268 & To AN-B (R19) & $\mathrm{X}$ & \\
\hline 1.20 & $2 \cdot \mathrm{in}:$ & $\begin{array}{l}\text { New diluent: } \\
\text { liner Y } 211\end{array}$ & $\begin{array}{l}\text { From blending lik-301 } \\
\text { (rew hoste delered) }\end{array}$ & $N A$ & Nat \\
\hline $\mathrm{L}-21$ & 3 -in. & $\begin{array}{l}\text { New diluent } \\
\text { line/W-211 }\end{array}$ & $\begin{array}{l}\text { From blending Tk-301 } \\
\text { (new nozzle) }\end{array}$ & $\mathrm{x}$ & \\
\hline
\end{tabular}


HNF-2854

Revision 0

Table 1. Nozzle Configuration Details. (7 Sheets)

\begin{tabular}{|c|c|c|c|c|c|}
\hline Nozzle & Size & Pipe \#/ Use & Configuration/Status & Jumper & Blank \\
\hline \multicolumn{6}{|c|}{ Location: Valve Pit AN-B Drawing No.: ES-314E-M40, Rev 7; H-14-20801; H-14-100946 } \\
\hline $\mathrm{R}-1$ & 3 -in. & Spare & $\begin{array}{l}\text { Isolation blank } \\
\text { installed }\end{array}$ & & $\mathrm{x}$ \\
\hline $\mathrm{R}-2$ & 2-in. & SN-260 & To AZ-02B (U7) & & $\mathrm{X}$ \\
\hline $\mathrm{R}-3$ & 2-in. & SL-160 & To AZ-02A (U12) & & $\mathrm{X}$ \\
\hline $\mathrm{R}-4$ & 2 -in. & Spare & $\begin{array}{l}\text { Isolation blank } \\
\text { installed }\end{array}$ & & $\mathrm{X}$ \\
\hline $\mathrm{R}-5$ & 2 -in. & SL-161 & To AN-01A (B) & $\mathrm{X}$ & \\
\hline $\mathrm{R}-7$ & 2-in. & SL-162 & To AN-02A (B) & $X$ & \\
\hline R-9 & 2-in. & SL-163 & To AN-03A (B) & $\mathrm{X}$ & \\
\hline $\mathrm{R}-10$ & 2-in. & Spare & $\begin{array}{l}\text { Isolation blank } \\
\text { installed }\end{array}$ & & $\mathrm{x}$ \\
\hline $\mathrm{R}-11$ & 3-in. & Spare & $\begin{array}{l}\begin{array}{l}\text { Isolation blank } \\
\text { installed }\end{array} \\
\end{array}$ & & $\mathrm{x}$ \\
\hline $\mathrm{R}-12$ & 3-in. & Spare & $\begin{array}{l}\text { Isolation blank } \\
\text { installed }\end{array}$ & & $\mathrm{x}$ \\
\hline$R-13$ & 3-in. & Spare & $\begin{array}{l}\text { Isolation blank } \\
\text { installed }\end{array}$ & & $\mathrm{X}$ \\
\hline R-14 & 3-in. & SN-263 & To AN-03A (A) & $\mathrm{x}$ & \\
\hline $\mathrm{R}-15$ & 3-in. & SN-261 & To $A N-01 A(A)$ & $\mathrm{X}$ & \\
\hline $\mathrm{R}-16$ & 3-in. & SN-262 & To AN-02A (A) & $\mathrm{X}$ & \\
\hline R-17 & 2-in. & Existing flush & Open & & $\mathrm{X}$ \\
\hline $\mathrm{R}-18$ & 2-in. & SL-168 & To AN-A (L18) & & $\mathrm{x}$ \\
\hline R-19 & 3-in. & SN-268 & \begin{tabular}{|l} 
To AN-A (L19) \\
\end{tabular} & $\mathrm{x}$ & \\
\hline $\mathrm{R}-20$ & 2-in. & $\begin{array}{l}\begin{array}{l}\text { New diluent } \\
\text { line/W-211 }\end{array} \\
\end{array}$ & $\begin{array}{l}\text { From blending Tk-301 } \\
\text { (new nozzle) }\end{array}$ & $\mathrm{X}$ & \\
\hline
\end{tabular}


HNF-2854

Revision 0

Table 1. Nozzle Configuration Details. (7 Sheets)

\begin{tabular}{|c|c|c|c|c|c|}
\hline Nozzle & Size & Pipe \#/ Use & Configuration/Status & Jumper & Blank \\
\hline \multicolumn{3}{|c|}{ Location: Valve Pit AY-01A } & \multicolumn{3}{|c|}{$\begin{array}{l}\text { Drawing No.: ES-314E-M40, Rev 7; H-2-70781; H-14- } \\
102646\end{array}$} \\
\hline U-1 & 3-in. & RW-3507 & Open & & $\mathrm{x}$ \\
\hline $\mathrm{U}-2$ & 2-in. & RW-3508 & Open & & $\mathrm{X}$ \\
\hline $\mathrm{U}-3$ & 4 -in. & Spare & Process blank installed & & $\mathrm{X}$ \\
\hline $\mathrm{U}-4$ & 4-in. & 6-in. PSW-D 501 & $\begin{array}{l}\text { Isolation blank } \\
\text { installed }\end{array}$ & & $\mathrm{X}$ \\
\hline U-5 & 3 -in. & PW-4550/4608 & $\begin{array}{l}\text { Isolation blank } \\
\text { installed }\end{array}$ & & $x$ \\
\hline U-6 & 3-in. & PW-4513 & $\begin{array}{l}\text { Isolation blank } \\
\text { installed }\end{array}$ & & $\mathrm{X}$ \\
\hline $\mathrm{U}-7$ & 1 -in. & RW-3509 & Not on routing board & & $\mathrm{X}$ \\
\hline U-8 & 2-in. & Spare & Open & & $\mathrm{X}$ \\
\hline $\mathrm{U}-9$ & 3 -in. & Spare & Not on routing board & & $\mathrm{X}$ \\
\hline U-10 & 2-in. & PW-4531 & Not on routing board & & $\mathrm{X}$ \\
\hline$U-12$ & 2-in. & SL-505 & Fr: AY-01D (U3) & $\mathrm{X}$ & \\
\hline $\mathrm{U}-13^{1}$ & 3-in. & New SN-635 & To AY-02A (U12) & $\mathrm{X}$ & \\
\hline Nozzle A & 2-in. & Slurry distributor & & $\mathrm{X}$ & \\
\hline \multicolumn{6}{|c|}{ Location: Valve Pit AY-02A } \\
\hline$U-1$ & 3 -in. & $\mathrm{RW}-3521$ & $\begin{array}{l}\text { Isolation blank } \\
\text { installed }\end{array}$ & & $\mathrm{x}$ \\
\hline $\mathrm{U}-2$ & 2 -in. & RW-3522 & $\begin{array}{l}\text { Isolation blank } \\
\text { installed }\end{array}$ & & $\mathrm{x}$ \\
\hline $\mathrm{U}-3$ & 4-in. & 2-in. SL-503 & Fr: AY-02D (U2) & $\mathrm{X}$ & \\
\hline U-4 & 4-in. & 6-in. PSW-D 502 & $\begin{array}{l}\text { Isolation blank } \\
\text { installed }\end{array}$ & & $\mathrm{X}$ \\
\hline U-5 & 3 -in. & New SN-633 & $\begin{array}{l}\text { To New AZ valve pit } \\
\text { Nozzle (TBD) }\end{array}$ & $\mathrm{X}$ & \\
\hline
\end{tabular}


HNF-2854

Revision 0

Table 1. Nozzle Configuration Details. (7 Sheets)

\begin{tabular}{|c|c|c|c|c|c|}
\hline Nozzle & Size & Pipe \#/ Use & Configuration/Status & Jumper & Blank \\
\hline U-6 & 3 -in. & PW-4519 & $\begin{array}{l}\text { Isolation blank } \\
\text { installed }\end{array}$ & & $\mathrm{X}$ \\
\hline $\mathrm{U}-7$ & 1 -in. & RW-3523 & Process blank installed & & $\mathrm{x}$ \\
\hline $\mathrm{U}-8$ & 2 -in. & SL-504 & To AY-01D (U2) & $\mathrm{X} / \mathrm{W}-320$ & \\
\hline U-10 & 2-in. & PW-4532 & $\begin{array}{l}\text { Isolation blank } \\
\text { installed }\end{array}$ & & $\mathrm{X}$ \\
\hline $\mathrm{U}-11$ & 4-in. & SL-100 & Fr: C-06A & $\mathrm{X} / \mathrm{W}-320$ & \\
\hline $\mathrm{U}-12$ & 3-in. & SN-635 & To AY-01A (U13) & $\mathrm{X}$ & \\
\hline \multicolumn{6}{|c|}{ Location: Valve Pit AZ-01A } \\
\hline $\mathrm{U}-2$ & 4-in. & PSW-4622 & $\begin{array}{l}\text { Isolation blank } \\
\text { installed }\end{array}$ & & $\mathrm{X}$ \\
\hline $\mathrm{U}-3$ & 2-in. & PW-4623 & Process blank installed & & $\mathrm{x}$ \\
\hline $\mathrm{U}-4$ & 4-in. & 6-in. PSW-D 603 & $\begin{array}{l}\text { Isolation blank } \\
\text { installed } \\
\end{array}$ & & $\mathrm{x}$ \\
\hline U-5 & 3-in. & PW-4607 & Open & & $\mathrm{x}$ \\
\hline $\mathrm{U}-6$ & 3 -in. & Spare & $\begin{array}{l}\text { Isolation blank } \\
\text { installed } \\
\end{array}$ & & $\mathrm{X}$ \\
\hline $\mathrm{U}-7$ & 4-in. & PSW-4621 & $\begin{array}{l}\text { Isolation blank } \\
\text { installed }\end{array}$ & & $\mathrm{x}$ \\
\hline $\mathrm{U}-8$ & 1 -in. & RW-3648 & AZ valve pit & & $\mathrm{x}$ \\
\hline U-9 & 4-in. & Spare & $\begin{array}{l}\text { Isolation blank } \\
\text { installed }\end{array}$ & & $\mathrm{X}$ \\
\hline $\mathrm{U}-10$ & 2-in. & SL-501 & Process blank installed & & $\mathrm{X}$ \\
\hline U-11 & 3 -in. & SN-631 & $\begin{array}{l}\text { To AZ-02A (U15) } \\
\text { (new nozzle) }\end{array}$ & $\mathrm{X} / \mathrm{W}-521$ & \\
\hline U-12 & 3 -in. & $\begin{array}{l}\text { New SN by } \\
\text { W-314 }\end{array}$ & $\begin{array}{l}\text { To "New AZ Valve } \\
\text { Pit"/(\# TBD) } \\
\text { (new nozzle) }\end{array}$ & $\mathrm{x}$ & \\
\hline
\end{tabular}


HNF-2854

Revision 0

Table 1. Nozzle Configuration Details. (7 Sheets)

\begin{tabular}{|c|c|c|c|c|c|}
\hline Nozzle & Size & Pipe \#/ Use & Configuration/Status & Jumper & Blank \\
\hline \multicolumn{6}{|c|}{ Location: Valve Pit AZ-02A Drawing No.: ES-314E-M40, Rev 7; H-2-70783 } \\
\hline $\mathrm{U}-2$ & 4-in. & PSW-S 607 & $\begin{array}{l}\text { Isolation blank } \\
\text { installed }\end{array}$ & & $\mathrm{x}$ \\
\hline U-3 & 2 -in. & PW-4609 & Process blank installed & & $\mathrm{X}$ \\
\hline $\mathrm{U}-4$ & 4-in. & 6-in. PSW-D 602 & To AZ-152 & $\mathrm{X}$ & \\
\hline U-5 & 3-in. & PW-4606 & $\begin{array}{l}\text { Isolation blank } \\
\text { installed }\end{array}$ & & $\mathrm{X}$ \\
\hline $\mathrm{U}-6$ & 3 -in. & Spare & Open & & $\mathrm{X}$ \\
\hline $\mathrm{U}-7$ & 4-in. & PSW-D 604 & Open & & $\mathrm{X}$ \\
\hline $\mathrm{U}-8$ & 1 -in. & Spare & Open & & $\mathrm{X}$ \\
\hline U-9 & 4-in. & Spare & Not on routing board & & $\mathrm{X}$ \\
\hline U-10 & 2-in. & SL-500 & Jumper AZ-02A (U12) & & $X$ \\
\hline U-11 & 2-in. & SL-501 & To AZ-01A (U10) & & $\mathrm{X}$ \\
\hline $\mathrm{U}-12$ & 2-in. & SL-160 & $\begin{array}{l}\text { Jumped AZ-02A } \\
\text { (U10) }\end{array}$ & & $\mathrm{X}$ \\
\hline U-13 & 3 -in. & $\begin{array}{l}\text { New SN-631 } \\
\text { W-314 }\end{array}$ & $\begin{array}{l}\text { To AZ-01A (U11) } \\
\text { (new nozzle) }\end{array}$ & $\mathrm{X}$ & \\
\hline U-14 & 3-in. & New flush line & $\begin{array}{l}\text { Fr: Blend Tk-301/ } \\
\text { W-211 (new nozzle) }\end{array}$ & $\begin{array}{c}\mathrm{X} \\
(\mathrm{W}-211)\end{array}$ & \\
\hline \multicolumn{6}{|c|}{ Location: Valve Pit AZ-02B ${ }^{2} \quad$ Drawing No.: ES-314E-M40, Rev 7; H-2-70782 } \\
\hline U-1 & 4-in. & 6-in. PSW-S 603 & Not on routing board & & $\mathrm{X}$ \\
\hline $\mathrm{U}-2$ & 4-in. & Spare & Not on routing board & & $\mathrm{X}$ \\
\hline U-3 & 4-in. & PSW-S 607 & Open & & $\mathrm{X}$ \\
\hline U-5 & 2-in. & SN-600 & Jump to AZ-02B (U-7) & & $\mathrm{X}$ \\
\hline U-6 & 2-in. & SN-601 & Open & & $\mathrm{x}$ \\
\hline U-7 & 2-in. & SN-260 & Jump to AZ-02B (U-5) & & $\mathrm{X}$ \\
\hline
\end{tabular}


HNF-2854

Revision 0

Table 1. Nozzle Configuration Details. (7 Sheets)

\begin{tabular}{|c|c|c|c|c|c|}
\hline Nozzle & Size & Pipe \#/ Use & Configuration/Status & Jumper & Blank \\
\hline \multicolumn{6}{|c|}{ Location: Valve Pit $\mathrm{AW}-\mathrm{A}^{3}$} \\
\hline L-1 & 3-in. & SN-267 & To AW-02A (J) & $\mathrm{x}$ & \\
\hline L-2 & 3-in. & SN-220 & To A-A valve pit (L2) & $\mathrm{X}$ & \\
\hline L-3 & 2-in. & SL-168 & To 242-A (18) & $\mathrm{X}$ & \\
\hline L-4 & 2-in. & SL-510 & To AP valve pit (2) & $\mathrm{X}$ & \\
\hline L-5 & 2-in. & Failed SL-161 & Open & & $\mathrm{X}$ \\
\hline L-7 & 2-in. & SL-163 & To AW-03A (B) & $\mathrm{X}$ & \\
\hline L-9 & 2-in. & SL-165 & To AW-05A (B) & $\mathrm{X}$ & \\
\hline $\mathrm{L}-10$ & 2 -in. & Spare & $\begin{array}{l}\text { Isolation blank } \\
\text { installed }\end{array}$ & & $\mathrm{x}$ \\
\hline L-11 & 3-in. & Spare & Open & & $\mathrm{X}$ \\
\hline $\mathrm{L}-12$ & 3-in. & P-V021 & Process blank installed & & $\mathrm{X}$ \\
\hline L-13 & 3 -in. & Spare & $\begin{array}{l}\text { Isolation blank } \\
\text { installed }\end{array}$ & & $\mathrm{X}$ \\
\hline $\mathrm{L}-14$ & 3-in. & SN-263 & To AW-03A (A) & $\mathrm{X}$ & \\
\hline L-15 & 3-in. & $\mathrm{SN}-265$ & To AW-05A (A) & $\mathrm{x}$ & \\
\hline L-16 & 3-in. & SN-261 & Process blank installed & $\mathrm{X}$ & \\
\hline $\mathrm{L}-17$ & 2-in. & Existing flush & Fr: AWFP-RW-V211 & & $\mathrm{X}$ \\
\hline $\mathrm{L}-18$ & 3 -in. & SL-169 & To AW-B (R18) & $\mathrm{X}$ & \\
\hline $\mathrm{L}-19$ & 3 -in. & SN-271 & To AW-B (R19) & $\mathrm{x}$ & \\
\hline \multicolumn{6}{|c|}{ Location: Valve Pit AW-B ${ }^{3} \quad$ Drawing No.: ES-314E-M40, Rev 7; H-14-20802 } \\
\hline R-1 & 3-in. & SN-268 & To AW-02A (H) & $\mathrm{X}$ & \\
\hline $\mathrm{R}-2$ & 3-in. & SN-219 & Process blank installed & & $\mathrm{X}$ \\
\hline $\mathrm{R}-3$ & 2-in. & SL-167 & To 242-A (19) & $\mathrm{X}$ & \\
\hline $\mathrm{R}-4$ & 2 -in. & SL-509 & To AP valve pit (1) & $\mathrm{X}$ & \\
\hline R-5 & 2 -in. & SL-162 & $\begin{array}{l}\text { Isolation blank } \\
\text { installed }\end{array}$ & & $\mathrm{x}$ \\
\hline
\end{tabular}


HNF-2854

Revision 0

Table 1. Nozzle Configuration Details. (7 Sheets)

\begin{tabular}{|c|c|c|c|c|c|}
\hline Nozzle & Size & Pipe \#/ Use & Configuration/Status & Jumper & Blank \\
\hline R-7 & 2 -in. & SL-164 & To AW-04A (B) & $\mathrm{x}$ & \\
\hline R-9 & 2 -in. & SL-166 & To AW-06A (B) & $\mathrm{x}$ & \\
\hline R-10 & 2-in. & Spare & $\begin{array}{l}\text { Isolation blank } \\
\text { installed }\end{array}$ & & $\mathrm{X}$ \\
\hline R-11 & 3 -in. & P-V023 & $\begin{array}{l}\text { Jumped to AW-B } \\
\text { (R12) }\end{array}$ & & $\mathrm{x}$ \\
\hline R-12 & 3-in. & P-V022 & $\begin{array}{l}\text { Jumped to AW-B } \\
\text { (R11) }\end{array}$ & & $\mathrm{x}$ \\
\hline $\mathrm{R}-13$ & 3 -in. & New flush line & Fr: Blending Tk-301 & $\mathrm{X}$ & \\
\hline R-14 & 3 -in. & SN-264 & To AW-04A (A) & $\mathrm{x}$ & \\
\hline $\mathrm{R}-15$ & 3 -in. & SN-266 & To AW-06A (A) & $\mathrm{x}$ & \\
\hline $\mathrm{R}-16$ & 3-in. & SN-262 & $\begin{array}{l}\text { Process blank installed } \\
\text { To AW-02A }\end{array}$ & $\mathrm{x}$ & \\
\hline R-17 & 2-in. & Existing flush & Fr: AWFP-RW-V-212 & $\mathrm{x}$ & \\
\hline R-18 & 2 -in. & SL-169 & To AW-A (L18) & $\mathrm{X}$ & \\
\hline R-19 & 3 -in. & SN-271 & To AW-A (L19) & $\mathrm{x}$ & \\
\hline $\mathrm{R}-20$ & 3-in. & $\begin{array}{l}\text { SN-247 (spare } \\
\text { line) }\end{array}$ & $\begin{array}{l}\text { Isolation blank } \\
\text { installed. To AW-04A }\end{array}$ & & $\mathrm{X}$ \\
\hline \multicolumn{6}{|c|}{ Location: Diversion Box A-151 Drawing No.: H-14-20802; H-14-20802 } \\
\hline 22 & Unk & To V004 & Process blank installed & & $\mathrm{X}$ \\
\hline 21 & Unk & To V005 & Process blank installed & & $\mathrm{X}$ \\
\hline 20 & Unk & To V006 & Process blank installed & & $\mathrm{X}$ \\
\hline $17,18,19$ & Unk & To V007 & Process blank installed & & $\mathrm{X}$ \\
\hline $14,15,16$ & Unk & To V008 & Process blank installed & & $\mathrm{x}$ \\
\hline
\end{tabular}

${ }^{1}$ Also connect AY-01A U-13 to existing slurry distributor assembly located in 42-in. riser.

${ }^{2} \mathrm{AZ}-02 \mathrm{~B}$ nozzle blanking is not within W-314 scope.

${ }^{3}$ Jumper connections and nozzles to be blanked currently are not the responsibility of

W-314. Project W-454 has the responsibility for providing the jumper configurations identified. 
HNF-2854

Revision 0

Table 2.Current Status. (5 Sheets)

\begin{tabular}{|c|c|c|c|c|}
\hline Location & Nozzle & Drawing \# & Use & Status \\
\hline \multicolumn{5}{|c|}{ AN-A Valve Pit } \\
\hline 241-AN-A & L-2 & H-14-020801 & Spare & Isolation blank installed \\
\hline 241-AN-A & $L-4$ & H-14-020801 & Spare & Isolation blank installed \\
\hline 241-AN-A & $L-10$ & H-14-020801 & Spare & Isolation blank installed \\
\hline 241-AN-A & L-11 & H-14-020801 & Spare & Isolation blank installed \\
\hline 241-AN-A & L-12 & H-14-020801 & Spare & Isolation blank installed \\
\hline 241-AN-A & $\mathrm{L}-13$ & H-14-020801 & Spare & Isolation blank installed \\
\hline 241-AN-A & L-17 & H-14-020801 & Existing flush & Process blank installed \\
\hline 241-AN-A & L-18 & H-14-020801 & To AN-B & $\begin{array}{l}\text { Rigid jumper installed (pit } \\
\text { crossover) }\end{array}$ \\
\hline 241-AN-A & $G$ & H-14-100944 & 3-in. jumper & *New nozzle to be installed \\
\hline 241-AN-A & C & H-14-100944 & 2-in. jumper & *New nozzle to be installed \\
\hline \multicolumn{5}{|c|}{ AN-B Valve Pit } \\
\hline 241-AN-B & R-1 & H-14-020801 & Spare & Isolation blank installed \\
\hline 241-AN-B & R-2 & H-14-020801 & $\begin{array}{l}\text { SN-260 to } \\
241-A Z-02 B\end{array}$ & Jumper installed \\
\hline 241-AN-B & R-3 & H-14-020801 & $\begin{array}{l}\text { SN-160 to } \\
241-A Z-02 A \\
\end{array}$ & Jumper installed \\
\hline 241-AN-B & R-4 & H-14-020801 & Spare & Isolation blank installed \\
\hline 241-AN-B & $\mathrm{R}-10$ & H-14-020801 & Spare & Isolation blank installed \\
\hline 241-AN-B & R-11 & H-14-020801 & Spare & Isolation blank installed \\
\hline 241-AN-B & R-12 & H-14-020801 & Spare & Isolation blank installed \\
\hline 241-AN-B & $\mathrm{R}-13$ & H-14-020801 & Spare & Isolation blank installed \\
\hline 241-AN-B & R-17 & H-14-020801 & Existing flush & Open \\
\hline 241-AN-B & R-18 & H-14-020801 & To 241-AN-B & $\begin{array}{l}\text { Rigid jumper installed (pit } \\
\text { crossover) }\end{array}$ \\
\hline 241-AN-B & E & H-14-100946 & 3-in. jumper & *New nozzle to be installed \\
\hline 241-AN-B & C & H-14-100946 & 2-in. jumper & *New nozzle to be installed \\
\hline
\end{tabular}


HNF-2854

Revision 0

Table 2.Current Status. (5 Sheets)

\begin{tabular}{|c|c|c|c|c|}
\hline Location & Nozzle & Drawing \# & Use & Status \\
\hline \multicolumn{5}{|c|}{ AY Process Pits } \\
\hline 241-AY-01A & U-1 & $\begin{array}{l}\text { H-2-070781 } \\
\text { H-14-102646 }\end{array}$ & $\begin{array}{l}3 \text {-in. RW from } \\
\text { RW valve pit }\end{array}$ & $\begin{array}{l}\text { *Not on routing board } \\
\text { *Open (pit video) } \\
\text { *Process blank installed }(\mathrm{H}-2)\end{array}$ \\
\hline 241-AY-01A & $\mathrm{U}-2$ & $\begin{array}{l}\text { H-2-070781 } \\
\text { H-14-102646 }\end{array}$ & $\begin{array}{l}\text { 2-in. RW from } \\
R W \text { valve pit }\end{array}$ & $\begin{array}{l}\text { *Rigid jumper installed (routing } \\
\text { board) } \\
{ }^{*} \text { Open (pit video) }\end{array}$ \\
\hline 241-AY-01A & $\mathrm{U}-4$ & \begin{tabular}{|l|l|}
$\mathrm{H}-2-070781$ \\
$\mathrm{H}-14-102646$ \\
\end{tabular} & $\begin{array}{l}\text { 6-in. PSW to } \\
\text { 241-AY-152 }\end{array}$ & Isolation blank installed \\
\hline 241-AY-01A & $\mathrm{U}-5$ & \begin{tabular}{|l|l|} 
H-2-070781 \\
H-14-102646 \\
\end{tabular} & $\begin{array}{l}\text { 3-in. PW to } \\
\text { 241-AY-152 }\end{array}$ & Isolation blank installed \\
\hline 241-AY-01A & U-6 & $\begin{array}{l}\mathrm{H}-2-070781 \\
\mathrm{H}-14-102646\end{array}$ & $\begin{array}{l}\text { 3-in. PW to } \\
\text { 241-AY-151 }\end{array}$ & Isolation blank installed \\
\hline 241-AY-01A & $\mathrm{U}-7$ & $\begin{array}{l}\text { H-2-070781 } \\
\text { H-14-102646 }\end{array}$ & $\begin{array}{l}1 \text {-in. RW from } \\
\text { RW valve pit }\end{array}$ & $\begin{array}{l}\text { *Not on routing board } \\
\text { *Process blank installed }(\mathrm{H}-2)\end{array}$ \\
\hline 241-AY-01A & U-8 & $\begin{array}{l}\text { H-2-070781 } \\
\text { H-14-102646 }\end{array}$ & Spare & $\begin{array}{l}\text { *Not on routing board } \\
\text { *Open (pit video) } \\
\text { *Dust cover installed (H-2) }\end{array}$ \\
\hline 241-AY-01A & U-9 & $\begin{array}{l}\text { H-2-070781 } \\
\text { H-14-102646 }\end{array}$ & Spare & $\begin{array}{l}\text { *Not on routing board } \\
\text { *Dust cover installed }(\mathrm{H}-2)\end{array}$ \\
\hline 241-AY-01A & U-10 & $\begin{array}{l}\text { H-2-070781 } \\
\text { H-14-102646 }\end{array}$ & $\begin{array}{l}\text { From 241-AY- } \\
01 \mathrm{~F}\end{array}$ & $\begin{array}{l}\text { *Not on routing board } \\
\text { *Process blank installed }(\mathrm{H}-2)\end{array}$ \\
\hline $241-\mathrm{AY}-02 \mathrm{~A}$ & U-1 & $\begin{array}{l}\text { H-2-070781 } \\
\text { H-2-100648 } \\
\text { H-2-818544 }\end{array}$ & $\begin{array}{l}\text { 3-in. RW from } \\
\text { RW valve pit }\end{array}$ & Isolation blank installed \\
\hline 241-AY-02A & U-2 & $\begin{array}{l}\text { H-2-070781 } \\
\text { H-2-100648 } \\
\text { H-2-818544 }\end{array}$ & $\begin{array}{l}\text { 2-in. RW from } \\
\text { RW valve pit }\end{array}$ & Isolation blank installed \\
\hline 241-AY-02A & U-4 & $\begin{array}{l}\text { H-2-070781 } \\
\text { H-2-100648 } \\
\text { H-2-818544 }\end{array}$ & $\begin{array}{l}\text { 6-in. PSW } \\
\text { from 241-AY- } \\
152\end{array}$ & Isolation blank installed \\
\hline $241-\mathrm{AY}-02 \mathrm{~A}$ & U-6 & $\begin{array}{l}\text { H-2-070781 } \\
\text { H-2-100648 } \\
\text { H-2-818544 }\end{array}$ & $\begin{array}{l}\text { 3-in. PW from } \\
241-A Y-151\end{array}$ & Isolation blank installed \\
\hline
\end{tabular}


HNF-2854

Revision 0

Table 2.Current Status. (5 Sheets)

\begin{tabular}{|c|c|c|c|c|}
\hline Location & Nozzle & Drawing \# & Use & Status \\
\hline 241-AY-02A & U-7 & $\begin{array}{l}\text { H-2-070781 } \\
\text { H-2-100648 } \\
\text { H-2-818544 }\end{array}$ & $\begin{array}{l}1 \text {-in. RW from } \\
\text { RW valve pit }\end{array}$ & Process blank installed \\
\hline 241-AY-02A & $\mathrm{U}-10$ & $\begin{array}{l}\text { H-2-070781 } \\
\text { H-2-100648 } \\
\text { H-2-818544 }\end{array}$ & $\begin{array}{l}\text { 2-in. PW from } \\
\text { 241-AY-02F }\end{array}$ & Isolation blank installed \\
\hline \multicolumn{5}{|c|}{ AZ Process Pits } \\
\hline 241-AZ-01A & $\mathrm{U}-2$ & H-2-070783 & $\begin{array}{l}\text { To 241-AZ- } \\
01 \mathrm{~B}\end{array}$ & $\begin{array}{l}\text { *Open (Routing board) } \\
\text { *Isolation blank installed (Pit } \\
\text { video) }\end{array}$ \\
\hline 241-AZ-01A & U-3 & H-2-070783 & $\begin{array}{l}\text { To 241-AZ- } \\
\text { 01F }\end{array}$ & Process blank installed \\
\hline 241-AZ-01A & U-4 & H-2-070783 & $\begin{array}{l}\text { 6-in. PSW to } \\
\text { 241-AZ-152 }\end{array}$ & $\begin{array}{l}\text { *Open (routing board) } \\
\text { *Isolation blank installed (Pit } \\
\text { video) }\end{array}$ \\
\hline 241-AZ-01A & U-5 & H-2-070783 & $\begin{array}{l}\text { 3-in. PW to } \\
241-A Z-152\end{array}$ & Open \\
\hline 241-AZ-01A & U-6 & H-2-070783 & Spare & $\begin{array}{l}\text { * Not on routing board } \\
\text { * Isolation blank installed (Pit } \\
\text { video) }\end{array}$ \\
\hline 241-AZ-01A & U-7 & $\mathrm{H}-2-070783$ & $\begin{array}{l}\text { To 241-AZ- } \\
01 \mathrm{C}\end{array}$ & $\begin{array}{l}\text { * Open (Routing board) } \\
\text { * Isolation blank installed (Pit } \\
\text { video) }\end{array}$ \\
\hline 241-AZ-01A & U-9 & H-2-070783 & Spare & $\begin{array}{l}\text { *Not on routing board } \\
\text { * Isolation blank installed (Pit } \\
\text { video) }\end{array}$ \\
\hline 241-AZ-01A & U-10 & H-2-070783 & SL-501 & Process blank installed \\
\hline 241-AZ-02A & $\mathrm{U}-2$ & $\mathrm{H}-2-070783$ & $\begin{array}{l}\text { To 241-AZ- } \\
\text { 02B }\end{array}$ & $\begin{array}{l}\text { *Process blank installed } \\
\text { (Routing board) } \\
\text { *Isolation blank installed (Pit } \\
\text { video) } \\
\end{array}$ \\
\hline 241-AZ-02A & U-3 & H-2-070783 & $\begin{array}{l}\text { To 241-AZ- } \\
02 \mathrm{~F}\end{array}$ & Process blank installed \\
\hline
\end{tabular}


HNF-2854

Revision 0

Table 2.Current Status. (5 Sheets)

\begin{tabular}{|c|c|c|c|c|}
\hline Location & Nozzle & Drawing \# & Use & Status \\
\hline 241-AZ-02A & U-5 & H-2-070783 & $\begin{array}{l}\text { To 241-AZ- } \\
152\end{array}$ & $\begin{array}{l}\text { *Open (Routing board) } \\
\text { *Isolation blank installed (Pit } \\
\text { video) }\end{array}$ \\
\hline 241-AZ-02A & U-6 & H-2-070783 & Spare & $\begin{array}{l}\text { *Not on routing board } \\
\text { *Open (Pit video) }\end{array}$ \\
\hline 241-AZ-02A & $\mathrm{U}-7$ & H-2-070783 & $\begin{array}{l}\text { To 241-AZ- } \\
02 \mathrm{C}\end{array}$ & Open \\
\hline 241-AZ-02A & U-8 & H-2-070783 & Spare & $\begin{array}{l}\text { *Not on routing board } \\
{ }^{*} \text { Open (Pit video) }\end{array}$ \\
\hline 241-AZ-02A & $\mathrm{U}-10$ & H-2-070783 & $\begin{array}{l}\text { SL-500 to } 241- \\
\text { AX-A }\end{array}$ & Rigid jumper installed \\
\hline 241-AZ-02A & U-12 & $\mathrm{H}-2-070783$ & $\begin{array}{l}\text { SL-160 to } 241- \\
\text { AN-B }\end{array}$ & Rigid jumper installed \\
\hline 241-AZ-02B & U-1 & H-2-070782 & $\begin{array}{l}\text { To 241-AZ- } \\
152 \\
\end{array}$ & $\begin{array}{l}\begin{array}{l}* \text { Not on routing board } \\
\text { *Process blank installed (H-2) }\end{array} \\
\end{array}$ \\
\hline 241-AZ-02B & $\mathrm{U}-2$ & H-2-070782 & Spare & $\begin{array}{l}* \text { Not on routing board } \\
* \text { Process blank installed }(\mathrm{H}-2)\end{array}$ \\
\hline 241-AZ-02B & U-3 & H-2-070782 & $\begin{array}{l}\text { To 241-AZ- } \\
02 \mathrm{~A}\end{array}$ & Open \\
\hline 241-AZ-02B & U-5 & H-2-070782 & $\begin{array}{l}\text { SN-600 to } \\
241-\mathrm{AX}-\mathrm{A} \\
\end{array}$ & Rigid jumper installed \\
\hline 241-AZ-02B & U-6 & H-2-070782 & $\begin{array}{l}\text { SN-601 to } \\
241-\mathrm{AZ}-01 \mathrm{C} \\
\end{array}$ & Open \\
\hline 241-AZ-02B & U-7 & H-2-070782 & $\begin{array}{l}\text { SN-260 to } \\
241-A N-B\end{array}$ & Rigid jumper installed \\
\hline \multicolumn{5}{|c|}{ AW-A Valve Pit } \\
\hline 241-AW-A & $\mathrm{L}-5$ & H-14-020802 & $\begin{array}{l}\text { Failed SL-161 } \\
\text { to 241-AW- } \\
01 \mathrm{~A}\end{array}$ & Open \\
\hline 241-AW-A & $\mathrm{L}-10$ & H-14-020802 & Spare & Isolation blank installed \\
\hline 241-AW-A & L-11 & H-14-020802 & Spare & Open \\
\hline
\end{tabular}


HNF-2854

Revision 0

Table 2.Current Status. (5 Sheets)

\begin{tabular}{|c|c|c|c|c|}
\hline Location & Nozzle & Drawing \# & Use & Status \\
\hline 241-AW-A & $\mathrm{L}-12$ & H-14-020802 & $\begin{array}{l}\text { From V021 - } \\
\text { PUREX }\end{array}$ & Process blank installed \\
\hline 241-AW-A & $\mathrm{L}-13$ & H-14-020802 & Spare & Isolation blank installed \\
\hline 241-AW-A & L-16 & H-14-020802 & $\begin{array}{l}\text { Existing flush } \\
\text { to } 241-\mathrm{AW} \text { - } \\
01 \mathrm{~A}\end{array}$ & Process blank installed \\
\hline \multicolumn{5}{|c|}{ AW-B Valve Pit } \\
\hline 241-AW-B & R-2 & H-14-020802 & $\begin{array}{l}\text { SN-219 to } \\
241-A-B\end{array}$ & Process blank installed \\
\hline 241-AW-B & R-5 & H-14-020802 & $\begin{array}{l}\text { SL-162 to } 241- \\
\text { AW-02A }\end{array}$ & Isolation blank installed \\
\hline 241-AW-B & $\mathbf{R}-10$ & H-14-020802 & Spare & Isolation blank installed \\
\hline 241-AW-B & R-11 & H-14-020802 & $\begin{array}{l}\text { V023 from } \\
\text { PUREX }\end{array}$ & Rigid jumper installed \\
\hline 241-AW-B & R-12 & H-14-020802 & $\begin{array}{l}\text { V022 from } \\
\text { PUREX }\end{array}$ & Rigid jumper installed \\
\hline 241-AW-B & R-16 & H-14-020802 & $\begin{array}{l}\text { Existing flush } \\
\text { to } 241 \text {-AW- } \\
02 \mathrm{~A}\end{array}$ & Process blank installed \\
\hline 241-AW-B & R-20 & H-14-020802 & $\begin{array}{l}\text { Spare to 241- } \\
\text { AW-04A }\end{array}$ & Isolation blank installed \\
\hline \multicolumn{5}{|c|}{ A-151 Diversion Box } \\
\hline $241-A-151$ & 22 & H-14-020802 & To V004 & Process blank installed \\
\hline $241-\mathrm{A}-151$ & 21 & H-14-020802 & To V005 & Process blank installed \\
\hline $241-A-151$ & 20 & H-14-020802 & To V006 & Process blank installed \\
\hline $241-A-151$ & $\begin{array}{l}17,18 \\
19\end{array}$ & H-14-020802 & To V007 & Process blank installed \\
\hline 241-A-151 & $\begin{array}{l}14,15, \\
16\end{array}$ & H-14-020802 & To V008 & Process blank installed \\
\hline
\end{tabular}

PUREX = Plutonium-Uranium Extraction

*Information from other sources. 
HNF-2854

Revision 0

This page intentionally left blank. 
DISTRIBUTION SHEET

\begin{tabular}{|c|c|c|c|c|c|}
\hline \multirow{2}{*}{$\begin{array}{l}\text { To } \\
\text { Distribution }\end{array}$} & \multirow{2}{*}{\multicolumn{3}{|c|}{$\begin{array}{l}\text { From } \\
\text { J. D. Galbraith, NHC }\end{array}$}} & \multicolumn{2}{|l|}{ Page 1 of 1} \\
\hline & & & & \multicolumn{2}{|c|}{ Date $6 / 16 / 98$} \\
\hline \multirow{2}{*}{\multicolumn{4}{|c|}{$\begin{array}{l}\text { Project Title/Work Order } \\
\text { Nozzle Evaluation for Project W-314, HNF-2854, Rev. O }\end{array}$}} & \multirow{2}{*}{\multicolumn{2}{|c|}{ EDT No. 622723}} \\
\hline & & & & & ECN No. \\
\hline Name & MSIN & $\begin{array}{l}\text { Text } \\
\text { With All } \\
\text { Attach. }\end{array}$ & Text Only & $\begin{array}{l}\text { Attach./ } \\
\text { Appendix } \\
\text { Only }\end{array}$ & $\begin{array}{l}\text { EDT/ECN } \\
\text { Only }\end{array}$ \\
\hline $\begin{array}{l}\text { Central Files } \\
\text { DOE Reading Room } \\
\text { DIMC (2) } \\
\text { Project W-314 File (2) }\end{array}$ & $\begin{array}{l}B 1-07 \\
H 2-53 \\
H 6-15 \\
R 1-29\end{array}$ & $\begin{array}{l}x \\
x \\
x \\
x\end{array}$ & & & \\
\hline $\begin{array}{l}\text { D. I. Allen } \\
\text { S. K. Baker } \\
\text { K. A. Boes } \\
\text { D. E. Bowers } \\
\text { R. A. Dodd } \\
\text { J. W. Fickl in } \\
\text { J. D. Galbraith } \\
\text { J. S. Garfield } \\
\text { J. L. Gilbert } \\
\text { J. L. Homan } \\
\text { R. W. Jacobson } \\
\text { D. L. McGrew } \\
\text { P. C. Miller } \\
\text { I. G. Papp } \\
\text { D. W. Reberger } \\
\text { M. D. Rickenbach } \\
\text { T. B. Salzano }\end{array}$ & $\begin{array}{l}\text { R2-50 } \\
\text { H5-49 } \\
\text { R3-25 } \\
\text { S5-13 } \\
\text { S5-07 } \\
\text { R2-88 } \\
\text { H5-49 } \\
\text { H5-49 } \\
\text { R3-47 } \\
\text { R3-25 } \\
\text { P8-47 } \\
\text { R3-25 } \\
\text { T4-08 } \\
\text { H5- } 49 \\
\text { S5-13 } \\
\text { G3-12 } \\
\text { G3-12 }\end{array}$ & $\begin{array}{l}x \\
x \\
x \\
x \\
x \\
x \\
x \\
x \\
x \\
x \\
x \\
x \\
x \\
x \\
x \\
x \\
x\end{array}$ & & & \\
\hline
\end{tabular}

